ANNALES

POLONICI MATHEMATICI

$92.1(2007)$

\title{
Linear liftings of symmetric tensor fields of type $(1,2)$ to Weil bundles
}

\author{
by JACEK DęBECKI (Kraków)
}

\begin{abstract}
This paper contains a classification of all linear liftings of symmetric tensor fields of type $(1,2)$ on $n$-dimensional manifolds to any tensor fields of type $(1,2)$ on Weil bundles under the condition that $n \geq 3$.
\end{abstract}

Introduction. Let $A$ be a Weil algebra inducing the Weil functor $T^{A}$ (see [3]) and let $n$ be a non-negative integer. We will denote by $M$ Te vector space of all tensor fields of type $(1,2)$ on a manifold $M$. Of course, $t \in \mathrm{Te} M$ is called symmetric if $t_{x}\left(y_{2}, y_{1}\right)=t_{x}\left(y_{1}, y_{2}\right)$ for every $x \in M$ and all $y_{1}, y_{2} \in T_{x} M$. The vector space of all symmetric tensor fields of type $(1,2)$ on $M$ will be denoted by SyTe $M$.

A lifting of symmetric tensor fields of type $(1,2)$ to tensor fields of type $(1,2)$ on $T^{A}$ is, by definition, a family of maps $L_{M}: \operatorname{SyTe} M \rightarrow \operatorname{Te} T^{A} M$ indexed by all $n$-dimensional manifolds and satisfying

$$
L_{M}\left(\phi^{*} t\right)=\left(T^{A} \phi\right)^{*}\left(L_{N}(t)\right)
$$

for all $n$-dimensional manifolds $M, N$, every embedding $\phi: M \rightarrow N$ and every $t \in \operatorname{SyTe} N$. Of course, here $\phi^{*} t$ is defined by the formula $\left(\phi^{*} t\right)_{x}=$ $\left(T_{x} \phi\right)^{-1} \circ t_{\phi(x)} \circ\left(T_{x} \phi, T_{x} \phi\right)$ for every $x \in M$. Such a lifting is said to be linear if $L_{M}$ is linear for every $n$-dimensional manifold $M$. Obviously, all linear liftings form a subspace of the vector space of all liftings in question.

Our purpose is to give a complete description of all these linear liftings. We hope that this result can be applied in the study of affine liftings of torsion-free linear connections to any linear connections on Weil bundles.

Construction of liftings. Our first task is to construct some liftings in question. We will use four known constructions which we now recall.

There is a unique lifting $C$ of tensor fields of type $(1,2)$ to tensor fields of type $(1,2)$ on $T^{A}$ such that $C_{U}^{i}(t)_{X}\left(Y_{1}, Y_{2}\right)=\left(T^{A} t_{j k}^{i}(X)\right) Y_{1}^{j} Y_{2}^{k}$ for every

2000 Mathematics Subject Classification: Primary 58A32.

Key words and phrases: natural operator, Weil bundle. 
$i \in\{1, \ldots, n\}$, every open subset $U$ of $\mathbb{R}^{n}$, every $t \in \mathrm{Te} U$, every $X \in T^{A} U$ and all $Y_{1}, Y_{2} \in A^{n}$ (see [2]).

For each $a \in A$ there is a unique natural tensor field $\widetilde{a}$ of type $(1,1)$ on $T^{A}$ such that $\left(\widetilde{a}_{U}^{i}\right)_{X}(Y)=a Y^{i}$ for every $i \in\{1, \ldots, n\}$, every open subset $U$ of $\mathbb{R}^{n}$, every $X \in T^{A} U$ and every $Y \in A^{n}$.

Let $C_{s} A$, where $s$ is a non-negative integer, denote the vector space of all $(s+1)$-linear maps $G: A \times \cdots \times A \rightarrow \mathbb{R}$ which are skew-symmetric with respect to the last $s$ variables and such that $G\left(a, b c, d_{2}, \ldots, d_{s}\right)=$ $G\left(a b, c, d_{2}, \ldots, d_{s}\right)+G\left(a c, b, d_{2}, \ldots, d_{s}\right)$ for all $a, b, c, d_{2}, \ldots, d_{s} \in A$ whenever $s \geq 1$. Note that elements of $C_{0} A$ are nothing but linear maps $A \rightarrow \mathbb{R}$. If $p, q$ are two non-negative integers such that $p \geq q$, then for each $G \in$ $C_{p-q} A$ there is a unique lifting $G^{p, q}$ of $p$-forms to $q$-forms on $T^{A}$ such that $G_{U}^{p, q}(\omega)_{X}\left(Y_{1}, \ldots, Y_{q}\right)=G\left(\left(T^{A} \omega_{i_{1} \ldots i_{p}}(X)\right) Y_{1}^{i_{1}} \ldots Y_{q}^{i_{q}}, X^{i_{q+1}}, \ldots, X^{i_{p}}\right)$ for every open subset $U$ of $\mathbb{R}^{n}$, every $p$-form $\omega$ on $U$, every $X \in T^{A} U$ and all $Y_{1}, \ldots, Y_{q} \in A^{n}$ (see [1]).

An $\mathbb{R}$-linear map $D: A \rightarrow A$ is said to be a derivation of $A$ if $D(a b)=$ $D(a) b+a D(b)$ for all $a, b \in A$. Let us denote by Der $A$ the vector space of all derivations of $A$. For each $D \in \operatorname{Der} A$ there is a unique natural vector field $\widetilde{D}$ on $T^{A}$ such that $\left(\widetilde{D}_{U}^{i}\right)_{X}=D\left(X^{i}\right)$ for every $i \in\{1, \ldots, n\}$, every open subset $U$ of $\mathbb{R}^{n}$ and every $X \in T^{A} U$ (see [3]).

We can now construct six types of linear liftings of symmetric tensor fields of type $(1,2)$ to tensor fields of type $(1,2)$ on $T^{A}$.

Let $P \in A$. Define $\bar{P}_{M}(t)=\widetilde{P} \circ C_{M}(t)$ for every $n$-dimensional manifold $M$ and every $t \in \operatorname{SyTe} M$. Thus $\bar{P}$ is the only lifting such that

$$
\bar{P}_{U}^{i}(t)_{X}\left(Y_{1}, Y_{2}\right)=P\left(T^{A} t_{j k}^{i}(X)\right) Y_{1}^{j} Y_{2}^{k}
$$

for every $i \in\{1, \ldots, n\}$, every open subset $U$ of $\mathbb{R}^{n}$, every $t \in$ SyTe $U$, every $X \in T^{A} U$ and all $Y_{1}, Y_{2} \in A^{n}$.

For every $n$-dimensional manifold $M$ and every $t \in$ Te $M$ we will denote by $\operatorname{tr} t$ the 1 -form on $M$ such that for every $x \in M$ and every $y \in T_{x} M$, $(\operatorname{tr} t)_{x}(y)$ is the trace of the endomorphism $T_{x} M \ni z \mapsto t_{x}(y, z) \in T_{x} M$. If $G \in C_{0} A$ and $a \in A$, then the formula $L_{M}(t)=G_{M}^{1,1}(\operatorname{tr} t) \otimes \widetilde{a}_{M}$ for every $n$-dimensional manifold $M$ and every $t \in \operatorname{SyTe} M$ defines a lifting $L$ we want. Since any sum of such liftings is also a lifting, we can carry out a more general construction. Let $Q \in C_{0} A \otimes A$. Clearly, $Q$ may be interpreted as an $\mathbb{R}$-linear map $A \rightarrow A$ and it is easy to see that there is a unique lifting $\bar{Q}$ such that

$$
\bar{Q}_{U}^{i}(t)_{X}\left(Y_{1}, Y_{2}\right)=Q\left(\left(T^{A} t_{k j}^{j}(X)\right) Y_{1}^{k}\right) Y_{2}^{i}
$$

for every $i \in\{1, \ldots, n\}$, every open subset $U$ of $\mathbb{R}^{n}$, every $t \in \operatorname{SyTe} U$, every $X \in T^{A} U$ and all $Y_{1}, Y_{2} \in A^{n}$. 
The last construction may be repeated with $\widetilde{a}_{M} \otimes G_{M}^{1,1}(\operatorname{tr} t)$ instead of $G_{M}^{1,1}(\operatorname{tr} t) \otimes \widetilde{a}_{M}$. Let $Q^{\prime} \in A \otimes C_{0} A$. Clearly, $Q^{\prime}$ may be interpreted as an $\mathbb{R}$-linear map $A \rightarrow A$ and it is easy to see that there is a unique lifting $\overline{Q^{\prime}}$ such that

$$
{\overline{Q^{\prime}}}_{U}^{i}(t)_{X}\left(Y_{1}, Y_{2}\right)=Q^{\prime}\left(\left(T^{A} t_{k j}^{j}(X)\right) Y_{2}^{k}\right) Y_{1}^{i}
$$

for every $i \in\{1, \ldots, n\}$, every open subset $U$ of $\mathbb{R}^{n}$, every $t \in \operatorname{SyTe} U$, every $X \in T^{A} U$ and all $Y_{1}, Y_{2} \in A^{n}$.

Let $d$ denote the exterior derivative. If $G \in C_{0} A$ and $D \in \operatorname{Der} A$, then the formula $L_{M}(t)=G_{M}^{2,2}(d(\operatorname{tr} t)) \otimes \widetilde{D}_{M}$ for every $n$-dimensional manifold $M$ and every $t \in$ SyTe $M$ defines a lifting $L$ we want. More generally, let $R \in$ $C_{0} A \otimes \operatorname{Der} A$. Clearly, $R$ may be interpreted as an $\mathbb{R}$-bilinear map $A \times A \rightarrow A$ with the property that $R(a, b c)=R(a, b) c+b R(a, c)$ for all $a, b, c \in A$ and it is easy to see that there is a unique lifting $\bar{R}$ such that

$$
\bar{R}_{U}^{i}(t)_{X}\left(Y_{1}, Y_{2}\right)=\frac{1}{2} R\left(\left(T^{A}\left(\frac{\partial t_{l j}^{j}}{\partial x^{k}}-\frac{\partial t_{k j}^{j}}{\partial x^{l}}\right)(X)\right) Y_{1}^{k} Y_{2}^{l}, X^{i}\right)
$$

for every $i \in\{1, \ldots, n\}$, every open subset $U$ of $\mathbb{R}^{n}$, every $t \in \operatorname{SyTe} U$, every $X \in T^{A} U$ and all $Y_{1}, Y_{2} \in A^{n}$.

If $G \in C_{1} A$ and $a \in A$, then the formula $L_{M}(t)=G_{M}^{2,1}(d(\operatorname{tr} t)) \otimes \widetilde{a}_{M}$ for every $n$-dimensional manifold $M$ and every $t \in$ SyTe $M$ defines a lifting $L$ we want. More generally, let $S \in C_{1} A \otimes A$. Clearly, $S$ may be interpreted as an $\mathbb{R}$ bilinear map $A \times A \rightarrow A$ with the property that $S(a, b c)=S(a b, c)+S(a c, b)$ for all $a, b, c \in A$ and it is easy to see that there is a unique lifting $\bar{S}$ such that

$$
\bar{S}_{U}^{i}(t)_{X}\left(Y_{1}, Y_{2}\right)=\frac{1}{2} S\left(\left(T^{A}\left(\frac{\partial t_{l j}^{j}}{\partial x^{k}}-\frac{\partial t_{k j}^{j}}{\partial x^{l}}\right)(X)\right) Y_{1}^{k}, X^{l}\right) Y_{2}^{i}
$$

for every $i \in\{1, \ldots, n\}$, every open subset $U$ of $\mathbb{R}^{n}$, every $t \in \operatorname{SyTe} U$, every $X \in T^{A} U$ and all $Y_{1}, Y_{2} \in A^{n}$.

The last construction may be repeated with $\widetilde{a}_{M} \otimes G_{M}^{2,1}(d(\operatorname{tr} t))$ instead of $G_{M}^{2,1}(d(\operatorname{tr} t)) \otimes \widetilde{a}_{M}$. Let $S^{\prime} \in A \otimes C_{1} A$. Clearly, $S^{\prime}$ may be interpreted as an $\mathbb{R}$-bilinear map $A \times A \rightarrow A$ with the property that $S^{\prime}(a, b c)=S^{\prime}(a b, c)+$ $S^{\prime}(a c, b)$ for all $a, b, c \in A$ and it is easy to see that there is a unique lifting $\overline{S^{\prime}}$ such that

$$
{\overline{S^{\prime}}}_{U}^{i}(t)_{X}\left(Y_{1}, Y_{2}\right)=\frac{1}{2} S^{\prime}\left(\left(T^{A}\left(\frac{\partial t_{l j}^{j}}{\partial x^{k}}-\frac{\partial t_{k j}^{j}}{\partial x^{l}}\right)(X)\right) Y_{2}^{k}, X^{l}\right) Y_{1}^{i}
$$

for every $i \in\{1, \ldots, n\}$, every open subset $U$ of $\mathbb{R}^{n}$, every $t \in \operatorname{SyTe} U$, every $X \in T^{A} U$ and all $Y_{1}, Y_{2} \in A^{n}$.

Classification theorem. We are now in a position to formulate our main result. 
THEOREM. If $n \geq 3$, then for each linear lifting $L$ of symmetric tensor fields of type $(1,2)$ to tensor fields of type $(1,2)$ on $T^{A}$ there are uniquely determined $P \in A, Q \in C_{0} A \otimes A, Q^{\prime} \in A \otimes C_{0} A, R \in C_{0} A \otimes \operatorname{Der} A$, $S \in C_{1} A \otimes A$ and $S^{\prime} \in A \otimes C_{1} A$ such that

$$
L=\bar{P}+\bar{Q}+\overline{Q^{\prime}}+\bar{R}+\bar{S}+\overline{S^{\prime}} .
$$

The remainder of the paper will be devoted to the proof of this theorem.

Lemma. We first prove an auxiliary result.

LEMMA. If $n \geq 2$ and $L, \widetilde{L}$ are two linear liftings of symmetric tensor fields of type $(1,2)$ to tensor fields of type $(1,2)$ on $T^{A}$, then

$$
L_{\mathbb{R}^{n}}\left(x^{2} \frac{\partial}{\partial x^{1}} \otimes d x^{1} \otimes d x^{1}\right)=\widetilde{L}_{\mathbb{R}^{n}}\left(x^{2} \frac{\partial}{\partial x^{1}} \otimes d x^{1} \otimes d x^{1}\right) \Rightarrow L=\widetilde{L} .
$$

Proof. It suffices to show that if

$$
L_{\mathbb{R}^{n}}\left(x^{2} \frac{\partial}{\partial x^{1}} \otimes d x^{1} \otimes d x^{1}\right)=0,
$$

then $L=0$.

From (8) and (1) with $\phi: \mathbb{R}^{n} \ni x \mapsto\left(x^{1}, x^{2}+1, x^{3}, \ldots, x^{n}\right) \in \mathbb{R}^{n}$ and $t=x^{2} \frac{\partial}{\partial x^{1}} \otimes d x^{1} \otimes d x^{1}$ it follows that

$$
L_{\mathbb{R}^{n}}\left(\frac{\partial}{\partial x^{1}} \otimes d x^{1} \otimes d x^{1}\right)=0,
$$

because $\phi^{*} t=\left(x^{2}+1\right) \frac{\partial}{\partial x^{1}} \otimes d x^{1} \otimes d x^{1}$.

From (9) and (1) with $\phi: \mathbb{R}^{n} \ni x \mapsto\left(x^{1}+\lambda x^{2}, x^{2}, \ldots, x^{n}\right) \in \mathbb{R}^{n}$, where $\lambda \in \mathbb{R}$, and $t=\frac{\partial}{\partial x^{1}} \otimes d x^{1} \otimes d x^{1}$ it follows that

$$
\begin{aligned}
L_{\mathbb{R}^{n}}\left(\frac{\partial}{\partial x^{1}} \otimes\left(d x^{1} \otimes d x^{2}+d x^{2} \otimes d x^{1}\right)\right) & =0, \\
L_{\mathbb{R}^{n}}\left(\frac{\partial}{\partial x^{1}} \otimes d x^{2} \otimes d x^{2}\right) & =0,
\end{aligned}
$$

because $\phi^{*} t=\frac{\partial}{\partial x^{1}} \otimes\left(d x^{1}+\lambda d x^{2}\right) \otimes\left(d x^{1}+\lambda d x^{2}\right)$ and because all coefficients of the zero polynomial are zero.

Let $\alpha \in \mathbb{N}^{n}$. Put $U=\left\{x \in \mathbb{R}^{n}: x^{1}>0, x^{2} \neq 0, \ldots, x^{n} \neq 0\right\}$ and $\psi: U \ni x \mapsto\left(\psi^{1}(x), x^{2}, \ldots, x^{n}\right) \in \mathbb{R}^{n}$, where

$$
\psi^{1}(x)= \begin{cases}\frac{\left(x^{1}\right)^{-\alpha^{1}+1}}{\left(-\alpha^{1}+1\right)\left(x^{2}\right)^{\alpha^{2}} \ldots\left(x^{n}\right)^{\alpha^{n}}} & \text { if } \alpha^{1} \neq 1, \\ \frac{\ln \left|x^{1}\right|}{\left(x^{2}\right)^{\alpha^{2}} \ldots\left(x^{n}\right)^{\alpha^{n}}} & \text { if } \alpha^{1}=1 .\end{cases}
$$


From (11) and (1) with $\psi$ instead of $\phi$ and with $t=\frac{\partial}{\partial x^{1}} \otimes d x^{2} \otimes d x^{2}$ it follows that $L_{U}\left(\left.x^{\alpha} \frac{\partial}{\partial x^{1}} \otimes d x^{2} \otimes d x^{2}\right|_{U}\right)=0$. Replacing $U$ by $V=\left\{x \in \mathbb{R}^{n}\right.$ : $\left.x^{1}<0, x^{2} \neq 0, \ldots, x^{n} \neq 0\right\}$ in the same manner we can see that $L_{V}\left(x^{\alpha} \frac{\partial}{\partial x^{1}} \otimes\right.$ $\left.\left.d x^{2} \otimes d x^{2}\right|_{V}\right)=0$. Since $U \cup V$ is a dense subset of $\mathbb{R}^{n}$,

$$
L_{\mathbb{R}^{n}}\left(x^{\alpha} \frac{\partial}{\partial x^{1}} \otimes d x^{2} \otimes d x^{2}\right)=0 .
$$

If $n \geq 3$, then (10) and (1) with $\phi: \mathbb{R}^{n} \ni x \mapsto\left(x^{1}+x^{3}, x^{2}, \ldots, x^{n}\right) \in \mathbb{R}^{n}$ and $t=\frac{\partial}{\partial x^{1}} \otimes\left(d x^{1} \otimes d x^{2}+d x^{2} \otimes d x^{1}\right)$ imply

$$
L_{\mathbb{R}^{n}}\left(\frac{\partial}{\partial x^{1}} \otimes\left(d x^{3} \otimes d x^{2}+d x^{2} \otimes d x^{3}\right)\right)=0,
$$

because $\phi^{*} t=\frac{\partial}{\partial x^{1}} \otimes\left(\left(d x^{1}+d x^{3}\right) \otimes d x^{2}+d x^{2} \otimes\left(d x^{1}+d x^{3}\right)\right)$.

If $n \geq 3$ and $\alpha \in \mathbb{N}^{n}$, then as in the proof of (12), from (13) and (1) with $\phi=\psi$ and $t=\frac{\partial}{\partial x^{1}} \otimes\left(d x^{3} \otimes d x^{2}+d x^{2} \otimes d x^{3}\right)$ it follows that

$$
L_{\mathbb{R}^{n}}\left(x^{\alpha} \frac{\partial}{\partial x^{1}} \otimes\left(d x^{3} \otimes d x^{2}+d x^{2} \otimes d x^{3}\right)\right)=0 .
$$

We now prove that for every $\alpha \in \mathbb{N}^{n}$,

$$
L_{\mathbb{R}^{n}}\left(x^{\alpha} \frac{\partial}{\partial x^{1}} \otimes d x^{1} \otimes d x^{1}\right)=0 .
$$

We consider two cases: $\alpha^{2} \neq 0$ and $\alpha^{2}=0$.

If $\alpha^{2} \neq 0$, then we obtain (15) from (8), (12) with $x^{1}$ and $x^{2}$ interchanged and (1) with $\phi: U \ni x \mapsto\left(x^{1}, x^{\alpha}, x^{3}, \ldots, x^{n}\right) \in \mathbb{R}^{n}$, where $U=$ $\left\{x \in \mathbb{R}^{n}: x^{1} \neq 0, x^{2}>0, x^{3} \neq 0, \ldots, x^{n} \neq 0\right\}$, and $t=x^{2} \frac{\partial}{\partial x^{1}} \otimes d x^{1} \otimes d x^{1}$, because $\phi^{*} t=\left.\left(x^{\alpha} \frac{\partial}{\partial x^{1}}-\frac{\alpha^{1}}{\alpha^{2}}\left(x^{1}\right)^{\alpha^{1}-1}\left(x^{2}\right)^{\alpha^{2}+1}\left(x^{3}\right)^{\alpha^{3}} \ldots\left(x^{n}\right)^{\alpha^{n}} \frac{\partial}{\partial x^{2}}\right) \otimes d x^{1} \otimes d x^{1}\right|_{U}$.

If $\alpha^{2}=0$, then we obtain (15) from (8), (12) with $x^{1}$ and $x^{2}$ interchanged and (1) with $\phi: \mathbb{R}^{n} \ni x \mapsto\left(x^{1}, x^{2}+x^{\alpha}, x^{3}, \ldots, x^{n}\right) \in \mathbb{R}^{n}$ and $t=x^{2} \frac{\partial}{\partial x^{1}} \otimes$ $d x^{1} \otimes d x^{1}$, because $\phi^{*} t=\left(x^{2}+x^{\alpha}\right)\left(\frac{\partial}{\partial x^{1}}-\alpha^{1}\left(x^{1}\right)^{\alpha^{1}-1}\left(x^{3}\right)^{\alpha^{3}} \ldots\left(x^{n}\right)^{\alpha^{n}} \frac{\partial}{\partial x^{2}}\right) \otimes$ $d x^{1} \otimes d x^{1}$.

From (8), (12) with $x^{1}$ and $x^{2}$ interchanged and (1) with $\phi: \mathbb{R}^{n} \ni x \mapsto$ $\left(x^{1}, x^{1}+x^{2}, x^{3}, \ldots, x^{n}\right) \in \mathbb{R}^{n}$ and $t=x^{2} \frac{\partial}{\partial x^{1}} \otimes d x^{1} \otimes d x^{1}$ it follows that

$$
L_{\mathbb{R}^{n}}\left(x^{1} \frac{\partial}{\partial x^{1}} \otimes d x^{1} \otimes d x^{1}\right)=0
$$

because $\phi^{*} t=\left(x^{1}+x^{2}\right)\left(\frac{\partial}{\partial x^{1}}-\frac{\partial}{\partial x^{2}}\right) \otimes d x^{1} \otimes d x^{1}$.

From (16), (8) and (1) with $\phi: \mathbb{R}^{n} \ni x \mapsto\left(x^{1}+\lambda x^{2}, x^{2}, \ldots, x^{n}\right) \in \mathbb{R}^{n}$, where $\lambda \in \mathbb{R}$, and $t=x^{1} \frac{\partial}{\partial x^{1}} \otimes d x^{1} \otimes d x^{1}$ it follows that

$$
L_{\mathbb{R}^{n}}\left(x^{1} \frac{\partial}{\partial x^{1}} \otimes\left(d x^{1} \otimes d x^{2}+d x^{2} \otimes d x^{1}\right)\right)=0,
$$

because $\phi^{*} t=\left(x^{1}+\lambda x^{2}\right) \frac{\partial}{\partial x^{1}} \otimes\left(d x^{1}+\lambda d x^{2}\right) \otimes\left(d x^{1}+\lambda d x^{2}\right)$. 
From (17) and (1) with $\phi: U \ni x \mapsto\left(x^{1}, \frac{1}{\alpha^{2}+1}\left(x^{2}\right)^{\alpha^{2}+1}, x^{3}, \ldots, x^{n}\right) \in \mathbb{R}^{n}$, where $U=\left\{x \in \mathbb{R}^{n}: x^{2}>0\right\}$ and $\alpha \in \mathbb{N}^{n}$, and $t=x^{1} \frac{\partial}{\partial x^{1}} \otimes\left(d x^{1} \otimes d x^{2}+\right.$ $\left.d x^{2} \otimes d x^{1}\right)$ it follows that

$$
L_{\mathbb{R}^{n}}\left(x^{1}\left(x^{2}\right)^{\alpha^{2}} \frac{\partial}{\partial x^{1}} \otimes\left(d x^{1} \otimes d x^{2}+d x^{2} \otimes d x^{1}\right)\right)=0 .
$$

We now prove that for every $\alpha \in \mathbb{N}^{n}$,

$$
L_{\mathbb{R}^{n}}\left(x^{\alpha} \frac{\partial}{\partial x^{1}} \otimes\left(d x^{1} \otimes d x^{2}+d x^{2} \otimes d x^{1}\right)\right)=0 .
$$

We consider two cases: $\alpha^{1} \neq 0$ and $\alpha^{1}=0$.

If $\alpha^{1} \neq 0$, then (19) can be deduced immediately from (18), (14), (14) with $x^{3}$ and $x^{i}$, where $i \in\{4, \ldots, n\}$, interchanged and (1) with $\phi: U \ni$ $x \mapsto\left(\left(x^{1}\right)^{\alpha^{1}}\left(x^{3}\right)^{\alpha^{3}} \ldots\left(x^{n}\right)^{\alpha^{n}}, x^{2}, \ldots, x^{n}\right) \in \mathbb{R}^{n}$, where $U=\left\{x \in \mathbb{R}^{n}: x^{1}>0\right.$, $\left.x^{3} \neq 0, \ldots, x^{n} \neq 0\right\}$, and $t=x^{1}\left(x^{2}\right)^{\alpha^{2}} \frac{\partial}{\partial x^{1}} \otimes\left(d x^{1} \otimes d x^{2}+d x^{2} \otimes d x^{1}\right)$, because $\phi^{*} t=\left.\frac{\partial}{\partial x^{1}} \otimes\left(\left(x^{\alpha} d x^{1}+\sum_{i=3}^{n} v_{i}(x) d x^{i}\right) \otimes d x^{2}+d x^{2} \otimes\left(x^{\alpha} d x^{1}+\sum_{i=3}^{n} v_{i}(x) d x^{i}\right)\right)\right|_{U}$, where $v_{i}(x)=\frac{\alpha^{i}}{\alpha^{1}}\left(x^{1}\right)^{\alpha^{1}+1}\left(x^{2}\right)^{\alpha^{2}} \ldots\left(x^{i-1}\right)^{\alpha^{i-1}}\left(x^{i}\right)^{\alpha^{i}-1}\left(x^{i+1}\right)^{\alpha^{i+1}} \ldots\left(x^{n}\right)^{\alpha^{n}}$ for every $i \in\{3, \ldots, n\}$.

If $\alpha^{1}=0$, then we obtain (19) from (17), (12), (14), (14) with $x^{3}$ and $x^{i}$, where $i \in\{4, \ldots, n\}$, interchanged and (1) with $\phi: \mathbb{R}^{n} \ni x \mapsto\left(x^{1}+x^{\alpha}\right.$, $\left.x^{2}, \ldots, x^{n}\right) \in \mathbb{R}^{n}$ and $t=x^{1} \frac{\partial}{\partial x^{1}} \otimes\left(d x^{1} \otimes d x^{2}+d x^{2} \otimes d x^{1}\right)$, because $\phi^{*} t=\left(x^{1}+x^{\alpha}\right) \frac{\partial}{\partial x^{1}} \otimes\left(\left(d x^{1}+\sum_{i=2}^{n} v_{i}(x) d x^{i}\right) \otimes d x^{2}+d x^{2} \otimes\left(d x^{1}+\sum_{i=2}^{n} v_{i}(x) d x^{i}\right)\right)$, where $v_{i}(x)=\alpha^{i}\left(x^{2}\right)^{\alpha^{2}} \ldots\left(x^{i-1}\right)^{\alpha^{i-1}}\left(x^{i}\right)^{\alpha^{i}-1}\left(x^{i+1}\right)^{\alpha^{i+1}} \ldots\left(x^{n}\right)^{\alpha^{n}}$ for every $i \in\{2, \ldots, n\}$.

Finally, we can take $\phi: \mathbb{R}^{n} \ni x \mapsto\left(x^{\sigma(1)}, \ldots, x^{\sigma(n)}\right) \in \mathbb{R}^{n}$, where $\sigma$ is any permutation of the set $\{1, \ldots, n\}$, in (1) to conclude from (12), (14), (15) and (19) that $L_{\mathbb{R}^{n}}\left(x^{\alpha} \frac{\partial}{\partial x^{i}} \otimes d x^{j} \otimes d x^{k}\right)=0$ for every $\alpha \in \mathbb{N}^{n}$ and all $i, j, k \in\{1, \ldots, n\}$. This forces $L_{\mathbb{R}^{n}}=0$ according to the Peetre theorem (see [3]), which proves the lemma.

Proof of the classification theorem. Fix a linear lifting $L$ of symmetric tensor fields of type $(1,2)$ to tensor fields of type $(1,2)$ on $T^{A}$. Clearly, for every $p \in\{1, \ldots, n\}$, every open subset $U$ of $\mathbb{R}^{n}$, every embedding $\phi: U \rightarrow \mathbb{R}^{n}$, every $t \in \operatorname{SyTe} \mathbb{R}^{n}$, every $X \in T^{A} U$ and all $Y_{1}, Y_{2} \in A^{n}$, 
condition (1) can be rewritten as

$$
\begin{aligned}
& \left(T^{A} \frac{\partial \phi^{p}}{\partial x^{q}}(X)\right) L_{U}^{q}\left(\phi^{*} t\right)_{X}\left(Y_{1}, Y_{2}\right) \\
& \quad=L_{\mathbb{R}^{n}}^{p}(t)_{T^{A} \phi(X)}\left(\left(T^{A} \frac{\partial \phi}{\partial x^{q}}(X)\right) Y_{1}^{q},\left(T^{A} \frac{\partial \phi}{\partial x^{q}}(X)\right) Y_{2}^{q}\right) .
\end{aligned}
$$

Formula (20) with $\phi: \mathbb{R}^{n} \ni x \mapsto\left(\lambda^{1} x^{1}, \ldots, \lambda^{n} x^{n}\right) \in \mathbb{R}^{n}$, where $\lambda^{1}, \ldots, \lambda^{n}$ $\in \mathbb{R} \backslash\{0\}$, and $t=x^{2} \frac{\partial}{\partial x^{1}} \otimes d x^{1} \otimes d x^{1}$ implies

$$
\lambda^{1} \lambda^{2} \lambda^{p} L_{\mathbb{R}^{n}}^{p}\left(x^{2} \frac{\partial}{\partial x^{1}} \otimes d x^{1} \otimes d x^{1}\right)_{X}\left(Y_{1}, Y_{2}\right)=
$$

$L_{\mathbb{R}^{n}}^{p}\left(x^{2} \frac{\partial}{\partial x^{1}} \otimes d x^{1} \otimes d x^{1}\right)_{\left(\lambda^{1} X^{1}, \ldots, \lambda^{n} X^{n}\right)}\left(\left(\lambda^{1} Y_{1}^{1}, \ldots, \lambda^{n} Y_{1}^{n}\right),\left(\lambda^{1} Y_{2}^{1}, \ldots, \lambda^{n} Y_{2}^{n}\right)\right)$.

By continuity, (21) still holds if $\lambda^{1}, \ldots, \lambda^{n} \in \mathbb{R}$. Using the homogeneous function theorem (see [3]) together with (21) and keeping in mind that for every $X \in A^{n}$ the map $L_{\mathbb{R}^{n}}\left(x^{2} \frac{\partial}{\partial x^{1}} \otimes d x^{1} \otimes d x^{1}\right)_{X}$ is $\mathbb{R}$-bilinear we deduce that there are unique $\mathbb{R}$-trilinear maps $a, b, c: A \times A \times A \rightarrow A$ such that

$$
\begin{aligned}
L_{\mathbb{R}^{n}}^{1}\left(x^{2} \frac{\partial}{\partial x^{1}} \otimes\right. & \left.d x^{1} \otimes d x^{1}\right)_{X}\left(Y_{1}, Y_{2}\right) \\
& =a\left(X^{1}, Y_{1}^{1}, Y_{2}^{2}\right)+b\left(X^{1}, Y_{1}^{2}, Y_{2}^{1}\right)+c\left(X^{2}, Y_{1}^{1}, Y_{2}^{1}\right),
\end{aligned}
$$

there are unique $\mathbb{R}$-trilinear maps $d, e, f: A \times A \times A \rightarrow A$ such that

$$
\begin{aligned}
L_{\mathbb{R}^{n}}^{2}\left(x^{2} \frac{\partial}{\partial x^{1}} \otimes\right. & \left.d x^{1} \otimes d x^{1}\right)_{X}\left(Y_{1}, Y_{2}\right) \\
& =d\left(X^{1}, Y_{1}^{2}, Y_{2}^{2}\right)+e\left(X^{2}, Y_{1}^{1}, Y_{2}^{2}\right)+f\left(X^{2}, Y_{1}^{2}, Y_{2}^{1}\right)
\end{aligned}
$$

and for every $p \in\{3, \ldots, n\}$ there are uniquely determined $\mathbb{R}$-trilinear maps $g^{p}, h^{p}, i^{p}, j^{p}, k^{p}, l^{p}: A \times A \times A \rightarrow A$ such that

$$
\begin{aligned}
& L_{\mathbb{R}^{n}}^{p}\left(x^{2} \frac{\partial}{\partial x^{1}} \otimes d x^{1} \otimes d x^{1}\right)_{X}\left(Y_{1}, Y_{2}\right)=g^{p}\left(X^{1}, Y_{1}^{2}, Y_{2}^{p}\right)+h^{p}\left(X^{1}, Y_{1}^{p}, Y_{2}^{2}\right) \\
+ & i^{p}\left(X^{2}, Y_{1}^{1}, Y_{2}^{p}\right)+j^{p}\left(X^{2}, Y_{1}^{p}, Y_{2}^{1}\right)+k^{p}\left(X^{p}, Y_{1}^{1}, Y_{2}^{2}\right)+l^{p}\left(X^{p}, Y_{1}^{2}, Y_{2}^{1}\right) .
\end{aligned}
$$

If $q \in\{4, \ldots, n\}$, then from (24) and (20) with $p=3, \phi: \mathbb{R}^{n} \ni x \mapsto$ $\left(x^{1}, x^{2}, x^{q}, x^{4}, \ldots, x^{q-1}, x^{3}, x^{q+1}, \ldots, x^{n}\right) \in \mathbb{R}^{n}$ and $t=x^{2} \frac{\partial}{\partial x^{1}} \otimes d x^{1} \otimes d x^{1}$ it follows that

$$
\begin{aligned}
g^{q}\left(X^{1}, Y_{1}^{2}, Y_{2}^{q}\right)+ & h^{q}\left(X^{1}, Y_{1}^{q}, Y_{2}^{2}\right)+i^{q}\left(X^{2}, Y_{1}^{1}, Y_{2}^{q}\right) \\
& +j^{q}\left(X^{2}, Y_{1}^{q}, Y_{2}^{1}\right)+k^{q}\left(X^{q}, Y_{1}^{1}, Y_{2}^{2}\right)+l^{q}\left(X^{q}, Y_{1}^{2}, Y_{2}^{1}\right) \\
= & g^{3}\left(X^{1}, Y_{1}^{2}, Y_{2}^{q}\right)+h^{3}\left(X^{1}, Y_{1}^{q}, Y_{2}^{2}\right)+i^{3}\left(X^{2}, Y_{1}^{1}, Y_{2}^{q}\right) \\
& +j^{3}\left(X^{2}, Y_{1}^{q}, Y_{2}^{1}\right)+k^{3}\left(X^{q}, Y_{1}^{1}, Y_{2}^{2}\right)+l^{3}\left(X^{q}, Y_{1}^{2}, Y_{2}^{1}\right) .
\end{aligned}
$$


Therefore $g^{q}=g^{3}, h^{q}=h^{3}, i^{q}=i^{3}, j^{q}=j^{3}, k^{q}=k^{3}, l^{q}=l^{3}$. Define $g=g^{3}$, $h=h^{3}, i=i^{3}, j=j^{3}, k=k^{3}, l=l^{3}$. Thus for every $p \in\{3, \ldots, n\}$, we can rewrite $(24)$ as

$$
\begin{aligned}
& L_{\mathbb{R}^{n}}^{p}\left(x^{2} \frac{\partial}{\partial x^{1}} \otimes d x^{1} \otimes d x^{1}\right)_{X}\left(Y_{1}, Y_{2}\right)=g\left(X^{1}, Y_{1}^{2}, Y_{2}^{p}\right)+h\left(X^{1}, Y_{1}^{p}, Y_{2}^{2}\right) \\
& \quad+i\left(X^{2}, Y_{1}^{1}, Y_{2}^{p}\right)+j\left(X^{2}, Y_{1}^{p}, Y_{2}^{1}\right)+k\left(X^{p}, Y_{1}^{1}, Y_{2}^{2}\right)+l\left(X^{p}, Y_{1}^{2}, Y_{2}^{1}\right) .
\end{aligned}
$$

From (25) and (20) with $p=3, U=\left\{x \in \mathbb{R}^{n}: x^{3}>0\right\}, \phi: U \ni x \mapsto$ $\left(x^{1}, x^{2},\left(x^{3}\right)^{2} / 2, x^{4}, \ldots, x^{n}\right) \in \mathbb{R}^{n}$ and $t=x^{2} \frac{\partial}{\partial x^{1}} \otimes d x^{1} \otimes d x^{1}$ it follows that

$$
\begin{aligned}
& X^{3}( g\left(X^{1}, Y_{1}^{2}, Y_{2}^{3}\right)+h\left(X^{1}, Y_{1}^{3}, Y_{2}^{2}\right)+i\left(X^{2}, Y_{1}^{1}, Y_{2}^{3}\right) \\
&\left.\quad+j\left(X^{2}, Y_{1}^{3}, Y_{2}^{1}\right)+k\left(X^{3}, Y_{1}^{1}, Y_{2}^{2}\right)+l\left(X^{3}, Y_{1}^{2}, Y_{2}^{1}\right)\right) \\
&=g\left(X^{1}, Y_{1}^{2}, X^{3} Y_{2}^{3}\right)+h\left(X^{1}, X^{3} Y_{1}^{3}, Y_{2}^{2}\right)+i\left(X^{2}, Y_{1}^{1}, X^{3} Y_{2}^{3}\right) \\
& \quad+j\left(X^{2}, X^{3} Y_{1}^{3}, Y_{2}^{1}\right)+k\left(\left(X^{3}\right)^{2} / 2, Y_{1}^{1}, Y_{2}^{2}\right)+l\left(\left(X^{3}\right)^{2} / 2, Y_{1}^{2}, Y_{2}^{1}\right)
\end{aligned}
$$

for every $X \in T^{A} U$. Replacing $U$ by $V=\left\{x \in \mathbb{R}^{n}: x^{3}<0\right\}$ in the same manner we can see that (26) holds for every $X \in T^{A} V$. Since $U \cup V$ is a dense subset of $\mathbb{R}^{n},(26)$ holds for every $X \in A^{n}$. Carrying out polarization if necessary we see from (26) that for all $w, x, y, z \in A$,

$$
\begin{gathered}
w g(x, y, z)=g(x, y, w z), \\
w h(x, y, z)=h(x, w y, z), \\
w i(x, y, z)=i(x, y, w z), \\
w j(x, y, z)=j(x, w y, z), \\
w k(x, y, z)+x k(w, y, z)=k(w x, y, z) .
\end{gathered}
$$

From (25) and (20) with $p=3, \phi: \mathbb{R}^{n} \ni x \mapsto\left(x^{1}, x^{2}+1, x^{3}, \ldots, x^{n}\right) \in \mathbb{R}^{n}$ and $t=x^{2} \frac{\partial}{\partial x^{1}} \otimes d x^{1} \otimes d x^{1}$ it follows that

$$
L_{\mathbb{R}^{n}}^{3}\left(\frac{\partial}{\partial x^{1}} \otimes d x^{1} \otimes d x^{1}\right)_{X}\left(Y_{1}, Y_{2}\right)=i\left(1, Y_{1}^{1}, Y_{2}^{3}\right)+j\left(1, Y_{1}^{3}, Y_{2}^{1}\right),
$$

because $\phi^{*} t=\left(x^{2}+1\right) \frac{\partial}{\partial x^{1}} \otimes d x^{1} \otimes d x^{1}$.

From (32) and (20) with $p=3, \phi: \mathbb{R}^{n} \ni x \mapsto\left(x^{1}+\lambda x^{2}, x^{2}, \ldots, x^{n}\right) \in \mathbb{R}^{n}$, where $\lambda \in \mathbb{R}$, and $t=\frac{\partial}{\partial x^{1}} \otimes d x^{1} \otimes d x^{1}$ it follows that

$$
L_{\mathbb{R}^{n}}^{3}\left(\frac{\partial}{\partial x^{1}} \otimes d x^{2} \otimes d x^{2}\right)_{X}\left(Y_{1}, Y_{2}\right)=0,
$$

because $\phi^{*} t=\frac{\partial}{\partial x^{1}} \otimes\left(d x^{1}+\lambda d x^{2}\right) \otimes\left(d x^{1}+\lambda d x^{2}\right)$ and because the respective coefficients of two equal polynomials are equal. 
From (33) and (20) with $p=3, U=\left\{x \in \mathbb{R}^{n}: x^{1} \neq 0\right\}, \phi: U \ni x \mapsto$ $\left(-1 / x^{1}, x^{2}, \ldots, x^{n}\right) \in \mathbb{R}^{n}$ and $t=\frac{\partial}{\partial x^{1}} \otimes d x^{2} \otimes d x^{2}$ it follows that

$$
L_{\mathbb{R}^{n}}^{3}\left(\left(x^{1}\right)^{2} \frac{\partial}{\partial x^{1}} \otimes d x^{2} \otimes d x^{2}\right)_{X}\left(Y_{1}, Y_{2}\right)=0 .
$$

From (34) and (20) with $p=3, \phi: \mathbb{R}^{n} \ni x \mapsto\left(x^{2}, x^{1}, x^{3}, \ldots, x^{n}\right) \in \mathbb{R}^{n}$ and $t=\left(x^{1}\right)^{2} \frac{\partial}{\partial x^{1}} \otimes d x^{2} \otimes d x^{2}$ it follows that

$$
L_{\mathbb{R}^{n}}^{3}\left(\left(x^{2}\right)^{2} \frac{\partial}{\partial x^{2}} \otimes d x^{1} \otimes d x^{1}\right)_{X}\left(Y_{1}, Y_{2}\right)=0 .
$$

From (25), (35) and (20) with $p=3, U=\left\{x \in \mathbb{R}^{n}: x^{1} \neq 0\right\}, \phi: U \ni$ $x \mapsto\left(x^{1}, x^{1} x^{2}, x^{3}, \ldots, x^{n}\right) \in \mathbb{R}^{n}$ and $t=x^{2} \frac{\partial}{\partial x^{1}} \otimes d x^{1} \otimes d x^{1}$ it follows that

$$
L_{\mathbb{R}^{n}}^{3}\left(x^{1} x^{2} \frac{\partial}{\partial x^{1}} \otimes d x^{1} \otimes d x^{1}\right)_{X}\left(Y_{1}, Y_{2}\right)
$$

$$
\begin{aligned}
= & g\left(X^{1}, X^{2} Y_{1}^{1}+X^{1} Y_{1}^{2}, Y_{2}^{3}\right)+h\left(X^{1}, Y_{1}^{3}, X^{2} Y_{2}^{1}+X^{1} Y_{2}^{2}\right)+i\left(X^{1} X^{2}, Y_{1}^{1}, Y_{2}^{3}\right) \\
& +j\left(X^{1} X^{2}, Y_{1}^{3}, Y_{2}^{1}\right)+k\left(X^{3}, Y_{1}^{1}, X^{2} Y_{2}^{1}+X^{1} Y_{2}^{2}\right)+l\left(X^{3}, X^{2} Y_{1}^{1}+X^{1} Y_{1}^{2}, Y_{2}^{1}\right),
\end{aligned}
$$

because $\phi^{*} t=\left.\left(x^{1} x^{2} \frac{\partial}{\partial x^{1}}-\left(x^{2}\right)^{2} \frac{\partial}{\partial x^{2}}\right) \otimes d x^{1} \otimes d x^{1}\right|_{U}$.

From (25) and (20) with $p=3, U=\left\{x \in \mathbb{R}^{n}: x^{1}>0\right\}, \phi: U \ni x \mapsto$ $\left(\left(x^{1}\right)^{2} / 2, x^{2}, \ldots, x^{n}\right) \in \mathbb{R}^{n}$ and $t=x^{2} \frac{\partial}{\partial x^{1}} \otimes d x^{1} \otimes d x^{1}$ it follows that

$$
\begin{aligned}
& L_{\mathbb{R}^{n}}^{3}\left(x^{1} x^{2} \frac{\partial}{\partial x^{1}} \otimes d x^{1} \otimes d x^{1}\right)_{X}\left(Y_{1}, Y_{2}\right) \\
&= g\left(\left(X^{1}\right)^{2} / 2, Y_{1}^{2}, Y_{2}^{3}\right)+h\left(\left(X^{1}\right)^{2} / 2, Y_{1}^{3}, Y_{2}^{2}\right)+i\left(X^{2}, X^{1} Y_{1}^{1}, Y_{2}^{3}\right) \\
& \quad+j\left(X^{2}, Y_{1}^{3}, X^{1} Y_{2}^{1}\right)+k\left(X^{3}, X^{1} Y_{1}^{1}, Y_{2}^{2}\right)+l\left(X^{3}, Y_{1}^{2}, X^{1} Y_{2}^{1}\right) .
\end{aligned}
$$

Carrying out polarization if necessary we see from (36) and (37) that for all $w, x, y, z \in A$,

$$
\begin{aligned}
g(w, x y, z)+g(x, w y, z) & =g(w x, y, z), \\
h(w, x, y z)+h(y, x, w z) & =h(w y, x, z), \\
g(w, x y, z)+i(w x, y, z) & =i(x, w y, z), \\
h(w, x, y z)+j(w y, x, z) & =j(y, x, w z), \\
k(w, x, y z) & =k(w, y x, z), \\
k(w, x, y z)+l(w, y x, z) & =0 .
\end{aligned}
$$

Define $R(y, z)=-2 k(z, y, 1)$ for all $y, z \in A$. According to (31), we have $x R(y, z)+z R(y, x)=R(y, x z)$ for all $x, y, z \in A$, and so $R \in C_{0} A \otimes \operatorname{Der} A$. By (5),

$$
\bar{R}_{\mathbb{R}^{n}}^{p}\left(x^{2} \frac{\partial}{\partial x^{1}} \otimes d x^{1} \otimes d x^{1}\right)_{X}\left(Y_{1}, Y_{2}\right)=\frac{1}{2} R\left(Y_{1}^{2} Y_{2}^{1}-Y_{1}^{1} Y_{2}^{2}, X^{p}\right) .
$$


From (42) it follows that $k(x, y, z)=-\frac{1}{2} R(z y, x)$ for all $x, y, z \in A$ and from (43) it follows that $l=-k$. Combining these with (44) gives, for every $p \in\{1, \ldots, n\}$,

(45) $\bar{R}_{\mathbb{R}^{n}}^{p}\left(x^{2} \frac{\partial}{\partial x^{1}} \otimes d x^{1} \otimes d x^{1}\right)_{X}\left(Y_{1}, Y_{2}\right)=k\left(X^{p}, Y_{1}^{1}, Y_{2}^{2}\right)+l\left(X^{p}, Y_{1}^{2}, Y_{2}^{1}\right)$.

Define $S(y, z)=2 g(z, y, 1)$ for all $y, z \in A$. According to (38), we have $S(x y, z)+S(z y, x)=S(y, z x)$ for all $x, y, z \in A$, and so $S \in C_{1} A \otimes A$. By (6),

$$
\bar{S}_{\mathbb{R}^{n}}^{p}\left(x^{2} \frac{\partial}{\partial x^{1}} \otimes d x^{1} \otimes d x^{1}\right)_{X}\left(Y_{1}, Y_{2}\right)=\frac{1}{2}\left(S\left(Y_{1}^{2}, X^{1}\right)-S\left(Y_{1}^{1}, X^{2}\right)\right) Y_{2}^{p} .
$$

From (27) it follows that $\frac{1}{2} x S(y, z)=g(z, y, x)$ for all $x, y, z \in A$. Combining this with (46) gives, for every $p \in\{1, \ldots, n\}$,

$$
\bar{S}_{\mathbb{R}^{n}}^{p}\left(x^{2} \frac{\partial}{\partial x^{1}} \otimes d x^{1} \otimes d x^{1}\right)_{X}\left(Y_{1}, Y_{2}\right)=g\left(X^{1}, Y_{1}^{2}, Y_{2}^{p}\right)-g\left(X^{2}, Y_{1}^{1}, Y_{2}^{p}\right) .
$$

Define $S^{\prime}(y, z)=2 h(z, 1, y)$ for all $y, z \in A$. According to (39), we have $S^{\prime}(x y, z)+S^{\prime}(z y, x)=S^{\prime}(y, z x)$ for all $x, y, z \in A$, and so $S^{\prime} \in A \otimes C_{1} A$. By (7),

$$
{\overline{S^{\prime}}}_{\mathbb{R}^{n}}^{p}\left(x^{2} \frac{\partial}{\partial x^{1}} \otimes d x^{1} \otimes d x^{1}\right)_{X}\left(Y_{1}, Y_{2}\right)=\frac{1}{2}\left(S^{\prime}\left(Y_{2}^{2}, X^{1}\right)-S^{\prime}\left(Y_{2}^{1}, X^{2}\right)\right) Y_{1}^{p}
$$

From (28) it follows that $\frac{1}{2} x S^{\prime}(y, z)=h(z, x, y)$ for all $x, y, z \in A$. Combining this with (48) gives, for every $p \in\{1, \ldots, n\}$,

$$
{\overline{S^{\prime}}}_{\mathbb{R}^{n}}^{p}\left(x^{2} \frac{\partial}{\partial x^{1}} \otimes d x^{1} \otimes d x^{1}\right)_{X}\left(Y_{1}, Y_{2}\right)=h\left(X^{1}, Y_{1}^{p}, Y_{2}^{2}\right)-h\left(X^{2}, Y_{1}^{p}, Y_{2}^{1}\right) .
$$

Put $L^{\prime}=L-\bar{R}-\bar{S}-\overline{S^{\prime}}$ as well as $a^{\prime}=a-k-h, b^{\prime}=b-l-g$, $c^{\prime}=c+g+h, d^{\prime}=d-g-h, e^{\prime}=e-k+g, f^{\prime}=f-l+h, i^{\prime}=i+g$ and $j^{\prime}=j+h$. From (45), (47) and (49) we see that (22), (23) and (25) lead to

$$
\begin{aligned}
L_{\mathbb{R}^{n}}^{\prime 1}\left(x^{2} \frac{\partial}{\partial x^{1}} \otimes\right. & \left.d x^{1} \otimes d x^{1}\right)_{X}\left(Y_{1}, Y_{2}\right) \\
& =a^{\prime}\left(X^{1}, Y_{1}^{1}, Y_{2}^{2}\right)+b^{\prime}\left(X^{1}, Y_{1}^{2}, Y_{2}^{1}\right)+c^{\prime}\left(X^{2}, Y_{1}^{1}, Y_{2}^{1}\right), \\
L_{\mathbb{R}^{n}}^{\prime 2}\left(x^{2} \frac{\partial}{\partial x^{1}}\right. & \left.\otimes d x^{1} \otimes d x^{1}\right)_{X}\left(Y_{1}, Y_{2}\right) \\
& =d^{\prime}\left(X^{1}, Y_{1}^{2}, Y_{2}^{2}\right)+e^{\prime}\left(X^{2}, Y_{1}^{1}, Y_{2}^{2}\right)+f^{\prime}\left(X^{2}, Y_{1}^{2}, Y_{2}^{1}\right), \\
L_{\mathbb{R}^{n}}^{\prime p}\left(x^{2} \frac{\partial}{\partial x^{1}} \otimes d x^{1} \otimes d x^{1}\right)_{X}\left(Y_{1}, Y_{2}\right) & =i^{\prime}\left(X^{2}, Y_{1}^{1}, Y_{2}^{p}\right)+j^{\prime}\left(X^{2}, Y_{1}^{p}, Y_{2}^{1}\right)
\end{aligned}
$$

for every $p \in\{3, \ldots, n\}$. Since (29), (30), (40) and (41) hold for every linear lifting satisfying (22), (23) and (25), upon comparing (22), (23) and (25) 
with (50), (51) and (52) we can assert that for all $w, x, y, z \in A$,

$$
\begin{aligned}
w i^{\prime}(x, y, z) & =i^{\prime}(x, y, w z), \\
w j^{\prime}(x, y, z) & =j^{\prime}(x, w y, z), \\
i^{\prime}(w x, y, z) & =i^{\prime}(x, w y, z), \\
j^{\prime}(w x, y, z) & =j^{\prime}(x, y, w z) .
\end{aligned}
$$

Define $Q(z)=i^{\prime}(z, 1,1)$ for every $z \in A$. Of course, $Q \in C_{0} A \otimes A$. By (3),

$$
\bar{Q}_{\mathbb{R}^{n}}^{p}\left(x^{2} \frac{\partial}{\partial x^{1}} \otimes d x^{1} \otimes d x^{1}\right)_{X}\left(Y_{1}, Y_{2}\right)=Q\left(X^{2} Y_{1}^{1}\right) Y_{2}^{p} .
$$

From (53) and (55) it follows that $x Q(y z)=i^{\prime}(z, y, x)$ for all $x, y, z \in A$. Combining this with (57) gives, for every $p \in\{1, \ldots, n\}$,

$$
\bar{Q}_{\mathbb{R}^{n}}^{p}\left(x^{2} \frac{\partial}{\partial x^{1}} \otimes d x^{1} \otimes d x^{1}\right)_{X}\left(Y_{1}, Y_{2}\right)=i^{\prime}\left(X^{2}, Y_{1}^{1}, Y_{2}^{p}\right) .
$$

Define $Q^{\prime}(z)=j^{\prime}(z, 1,1)$ for every $z \in A$. Of course, $Q^{\prime} \in A \otimes C_{0} A$. By (4),

$$
{\overline{Q^{\prime}}}_{\mathbb{R}^{n}}^{p}\left(x^{2} \frac{\partial}{\partial x^{1}} \otimes d x^{1} \otimes d x^{1}\right)_{X}\left(Y_{1}, Y_{2}\right)=Q^{\prime}\left(X^{2} Y_{2}^{1}\right) Y_{1}^{p}
$$

From (54) and (56) it follows that $x Q^{\prime}(y z)=j^{\prime}(z, x, y)$ for all $x, y, z \in A$. Combining this with (59) gives, for every $p \in\{1, \ldots, n\}$,

$$
{\overline{Q^{\prime}}}_{\mathbb{R}^{n}}^{p}\left(x^{2} \frac{\partial}{\partial x^{1}} \otimes d x^{1} \otimes d x^{1}\right)_{X}\left(Y_{1}, Y_{2}\right)=j^{\prime}\left(X^{2}, Y_{1}^{p}, Y_{2}^{1}\right) .
$$

Put $L^{\prime \prime}=L^{\prime}-\bar{Q}-\overline{Q^{\prime}}$ as well as $a^{\prime \prime}=a^{\prime}, b^{\prime \prime}=b^{\prime}, c^{\prime \prime}=c^{\prime}-i^{\prime}-j^{\prime}, d^{\prime \prime}=d^{\prime}$, $e^{\prime \prime}=e^{\prime}-i^{\prime}$ and $f^{\prime \prime}=f^{\prime}-j^{\prime}$. From (58) and (60) we deduce that (50), (51) and (52) lead to

$$
\begin{aligned}
& L_{\mathbb{R}^{n}}^{\prime \prime 1}\left(x^{2} \frac{\partial}{\partial x^{1}} \otimes d x^{1} \otimes d x^{1}\right)_{X}\left(Y_{1}, Y_{2}\right) \\
& =a^{\prime \prime}\left(X^{1}, Y_{1}^{1}, Y_{2}^{2}\right)+b^{\prime \prime}\left(X^{1}, Y_{1}^{2}, Y_{2}^{1}\right)+c^{\prime \prime}\left(X^{2}, Y_{1}^{1}, Y_{2}^{1}\right) \text {, } \\
& \text { (62) } L_{\mathbb{R}^{n}}^{\prime \prime 2}\left(x^{2} \frac{\partial}{\partial x^{1}} \otimes d x^{1} \otimes d x^{1}\right)_{X}\left(Y_{1}, Y_{2}\right) \\
& =d^{\prime \prime}\left(X^{1}, Y_{1}^{2}, Y_{2}^{2}\right)+e^{\prime \prime}\left(X^{2}, Y_{1}^{1}, Y_{2}^{2}\right)+f^{\prime \prime}\left(X^{2}, Y_{1}^{2}, Y_{2}^{1}\right) \text {, } \\
& \text { (63) } L_{\mathbb{R}^{n}}^{\prime \prime p}\left(x^{2} \frac{\partial}{\partial x^{1}} \otimes d x^{1} \otimes d x^{1}\right)_{X}\left(Y_{1}, Y_{2}\right)=0
\end{aligned}
$$

for every $p \in\{3, \ldots, n\}$. 
From (63) and (20) with $p=3, \phi: \mathbb{R}^{n} \ni x \mapsto\left(x^{1}, x^{2}+x^{3}, x^{3}, \ldots, x^{n}\right) \in$ $\mathbb{R}^{n}$ and $t=x^{2} \frac{\partial}{\partial x^{1}} \otimes d x^{1} \otimes d x^{1}$ it follows that

$$
L_{\mathbb{R}^{n}}^{\prime \prime 3}\left(x^{3} \frac{\partial}{\partial x^{1}} \otimes d x^{1} \otimes d x^{1}\right)_{X}\left(Y_{1}, Y_{2}\right)=0
$$

because $\phi^{*} t=\left(x^{2}+x^{3}\right) \frac{\partial}{\partial x^{1}} \otimes d x^{1} \otimes d x^{1}$.

From (62) and (20) with $p=2, \phi: \mathbb{R}^{n} \ni x \mapsto\left(x^{1}, x^{3}, x^{2}, x^{4}, \ldots, x^{n}\right) \in \mathbb{R}^{n}$ and $t=x^{2} \frac{\partial}{\partial x^{1}} \otimes d x^{1} \otimes d x^{1}$ it follows that

$$
\begin{aligned}
L_{\mathbb{R}^{n}}^{\prime \prime 3}\left(x^{3} \frac{\partial}{\partial x^{1}}\right. & \left.\otimes d x^{1} \otimes d x^{1}\right)_{X}\left(Y_{1}, Y_{2}\right) \\
& =d^{\prime \prime}\left(X^{1}, Y_{1}^{3}, Y_{2}^{3}\right)+e^{\prime \prime}\left(X^{3}, Y_{1}^{1}, Y_{2}^{3}\right)+f^{\prime \prime}\left(X^{3}, Y_{1}^{3}, Y_{2}^{1}\right) .
\end{aligned}
$$

Comparing (64) with (65) we see that $d^{\prime \prime}=0, e^{\prime \prime}=0$ and $f^{\prime \prime}=0$, which enables us to rewrite (62) as

$$
L_{\mathbb{R}^{n}}^{\prime \prime 2}\left(x^{2} \frac{\partial}{\partial x^{1}} \otimes d x^{1} \otimes d x^{1}\right)_{X}\left(Y_{1}, Y_{2}\right)=0
$$

If $\phi: \mathbb{R}^{n} \ni x \mapsto\left(x^{1}, x^{2}+1, x^{3}, \ldots, x^{n}\right) \in \mathbb{R}^{n}$ and $t=x^{2} \frac{\partial}{\partial x^{1}} \otimes d x^{1} \otimes d x^{1}$, then $\phi^{*} t=\left(x^{2}+1\right) \frac{\partial}{\partial x^{1}} \otimes d x^{1} \otimes d x^{1}$. Taking successively $p=1,2,3$ and these $\phi$ and $t$ in (20) and combining this with (61), (66) and (63) respectively we get

$$
\begin{aligned}
& L_{\mathbb{R}^{n}}^{\prime \prime 1}\left(\frac{\partial}{\partial x^{1}} \otimes d x^{1} \otimes d x^{1}\right)_{X}\left(Y_{1}, Y_{2}\right)=c^{\prime \prime}\left(1, Y_{1}^{1}, Y_{2}^{1}\right), \\
& L_{\mathbb{R}^{n}}^{\prime \prime 2}\left(\frac{\partial}{\partial x^{1}} \otimes d x^{1} \otimes d x^{1}\right)_{X}\left(Y_{1}, Y_{2}\right)=0, \\
& L_{\mathbb{R}^{n}}^{\prime \prime 3}\left(\frac{\partial}{\partial x^{1}} \otimes d x^{1} \otimes d x^{1}\right)_{X}\left(Y_{1}, Y_{2}\right)=0 .
\end{aligned}
$$

If $\phi: \mathbb{R}^{n} \ni x \mapsto\left(x^{1}+\lambda x^{2}, x^{2}, \ldots, x^{n}\right) \in \mathbb{R}^{n}$, where $\lambda \in \mathbb{R}$, and $t=$ $\frac{\partial}{\partial x^{1}} \otimes d x^{1} \otimes d x^{1}$, then $\phi^{*} t=\frac{\partial}{\partial x^{1}} \otimes\left(d x^{1}+\lambda d x^{2}\right) \otimes\left(d x^{1}+\lambda d x^{2}\right)$. Upon taking $p=1,2,3$ (2 before 1 ) and these $\phi$ and $t$ in (20) and combining this with (67), (68) and (69) respectively we get

$$
\begin{aligned}
& L_{\mathbb{R}^{n}}^{\prime \prime 1}\left(\frac{\partial}{\partial x^{1}} \otimes d x^{2} \otimes d x^{2}\right)_{X}\left(Y_{1}, Y_{2}\right)=c^{\prime \prime}\left(1, Y_{1}^{2}, Y_{2}^{2}\right), \\
& L_{\mathbb{R}^{n}}^{\prime \prime 2}\left(\frac{\partial}{\partial x^{1}} \otimes d x^{2} \otimes d x^{2}\right)_{X}\left(Y_{1}, Y_{2}\right)=0, \\
& L_{\mathbb{R}^{n}}^{\prime \prime 3}\left(\frac{\partial}{\partial x^{1}} \otimes d x^{2} \otimes d x^{2}\right)_{X}\left(Y_{1}, Y_{2}\right)=0 .
\end{aligned}
$$

From (70) and (20) with $p=1, U=\left\{x \in \mathbb{R}^{n}: x^{1}>0\right\}, \phi: U \ni x \mapsto$ $\left(\ln \left|x^{1}\right|, x^{2}, \ldots, x^{n}\right) \in \mathbb{R}^{n}$ and $t=\frac{\partial}{\partial x^{1}} \otimes d x^{2} \otimes d x^{2}$ it follows that 


$$
L_{\mathbb{R}^{n}}^{\prime \prime 1}\left(x^{1} \frac{\partial}{\partial x^{1}} \otimes d x^{2} \otimes d x^{2}\right)_{X}\left(Y_{1}, Y_{2}\right)=X^{1} c^{\prime \prime}\left(1, Y_{1}^{2}, Y_{2}^{2}\right) .
$$

From (73) and (20) with $p=1, \phi: \mathbb{R}^{n} \ni x \mapsto\left(x^{2}, x^{1}, x^{3}, \ldots, x^{n}\right) \in \mathbb{R}^{n}$ and $t=x^{1} \frac{\partial}{\partial x^{1}} \otimes d x^{2} \otimes d x^{2}$ it follows that

$$
L_{\mathbb{R}^{n}}^{\prime \prime 2}\left(x^{2} \frac{\partial}{\partial x^{2}} \otimes d x^{1} \otimes d x^{1}\right)_{X}\left(Y_{1}, Y_{2}\right)=X^{2} c^{\prime \prime}\left(1, Y_{1}^{1}, Y_{2}^{1}\right) .
$$

From (61), (74), (66) and (20) with $p=2, \phi: \mathbb{R}^{n} \ni x \mapsto\left(x^{1}, \lambda x^{1}+x^{2}\right.$, $\left.x^{3}, \ldots, x^{n}\right) \in \mathbb{R}^{n}$, where $\lambda \in \mathbb{R}$, and $t=x^{2} \frac{\partial}{\partial x^{1}} \otimes d x^{1} \otimes d x^{1}$ it follows that

$$
\begin{aligned}
& L_{\mathbb{R}^{n}}^{\prime \prime 2}\left(x^{1} \frac{\partial}{\partial x^{1}} \otimes d x^{1} \otimes d x^{1}\right)_{X}\left(Y_{1}, Y_{2}\right) \\
= & -a^{\prime \prime}\left(X^{1}, Y_{1}^{1}, Y_{2}^{2}\right)-b^{\prime \prime}\left(X^{1}, Y_{1}^{2}, Y_{2}^{1}\right)-c^{\prime \prime}\left(X^{2}, Y_{1}^{1}, Y_{2}^{1}\right)+X^{2} c^{\prime \prime}\left(1, Y_{1}^{1}, Y_{2}^{1}\right),
\end{aligned}
$$

because $\phi^{*} t=\left(\lambda x^{1}+x^{2}\right)\left(\frac{\partial}{\partial x^{1}}-\lambda \frac{\partial}{\partial x^{2}}\right) \otimes d x^{1} \otimes d x^{1}$.

From (68) and (20) with $p=2, U=\left\{x \in \mathbb{R}^{n}: x^{1}>0\right\}, \phi: U \ni x \mapsto$ $\left(\left(x^{1}\right)^{2} / 2, x^{2}, \ldots, x^{n}\right) \in \mathbb{R}^{n}$ and $t=\frac{\partial}{\partial x^{1}} \otimes d x^{1} \otimes d x^{1}$ it follows that

$$
L_{\mathbb{R}^{n}}^{\prime \prime 2}\left(x^{1} \frac{\partial}{\partial x^{1}} \otimes d x^{1} \otimes d x^{1}\right)_{X}\left(Y_{1}, Y_{2}\right)=0 .
$$

Comparing (75) with (76) we see that $a^{\prime \prime}=0$ and $b^{\prime \prime}=0$, which enables us to rewrite (61) as

$$
L_{\mathbb{R}^{n}}^{\prime \prime 1}\left(x^{2} \frac{\partial}{\partial x^{1}} \otimes d x^{1} \otimes d x^{1}\right)_{X}\left(Y_{1}, Y_{2}\right)=c^{\prime \prime}\left(X^{2}, Y_{1}^{1}, Y_{2}^{1}\right),
$$

and that for all $x, y, z \in A$,

$$
x c^{\prime \prime}(1, y, z)=c^{\prime \prime}(x, y, z) .
$$

If $\phi: \mathbb{R}^{n} \ni x \mapsto\left(x^{1}, x^{2}+\lambda x^{3}, x^{3}, \ldots, x^{n}\right) \in \mathbb{R}^{n}$, where $\lambda \in \mathbb{R}$, and $t=\frac{\partial}{\partial x^{1}} \otimes d x^{2} \otimes d x^{2}$, then $\phi^{*} t=\frac{\partial}{\partial x^{1}} \otimes\left(d x^{2}+\lambda d x^{3}\right) \otimes\left(d x^{2}+\lambda d x^{3}\right)$. Upon taking $p=1,2,3$ (3 before 2) and these $\phi$ and $t$ in (20) and combining this with (70), (71) and (72) respectively we get

$$
\begin{aligned}
L_{\mathbb{R}^{n}}^{\prime \prime 1}\left(\frac { \partial } { \partial x ^ { 1 } } \otimes \left(d x^{2} \otimes d x^{3}+d x^{3} \otimes\right.\right. & \left.\left.d x^{2}\right)\right)_{X}\left(Y_{1}, Y_{2}\right) \\
& =c^{\prime \prime}\left(1, Y_{1}^{2}, Y_{2}^{3}\right)+c^{\prime \prime}\left(1, Y_{1}^{3}, Y_{2}^{2}\right),
\end{aligned}
$$

(80) $\quad L_{\mathbb{R}^{n}}^{\prime \prime 2}\left(\frac{\partial}{\partial x^{1}} \otimes\left(d x^{2} \otimes d x^{3}+d x^{3} \otimes d x^{2}\right)\right)_{X}\left(Y_{1}, Y_{2}\right)=0$

$$
L_{\mathbb{R}^{n}}^{\prime \prime 3}\left(\frac{\partial}{\partial x^{1}} \otimes\left(d x^{2} \otimes d x^{3}+d x^{3} \otimes d x^{2}\right)\right)_{X}\left(Y_{1}, Y_{2}\right)=0 .
$$

If $U=\left\{x \in \mathbb{R}^{n}: 1+x^{2} x^{3} \neq 0\right\}, \phi: U \ni x \mapsto\left(\frac{x^{1}}{1+x^{2} x^{3}}, x^{2}, \ldots, x^{n}\right) \in \mathbb{R}^{n}$ and $t=\frac{\partial}{\partial x^{1}} \otimes\left(d x^{2} \otimes d x^{3}+d x^{3} \otimes d x^{2}\right)$, then $\phi^{*} t=\left(1+x^{2} x^{3}\right) \frac{\partial}{\partial x^{1}} \otimes\left(d x^{2} \otimes d x^{3}\right.$ 
$\left.+d x^{3} \otimes d x^{2}\right)\left.\right|_{U}$. Taking $p=1,2,3$ (2 and 3 before 1$)$ and these $\phi$ and $t$ in (20) and combining this with (79), (80) and (81) respectively we get

$$
\begin{aligned}
L_{\mathbb{R}^{n}}^{\prime \prime 1}\left(x ^ { 2 } x ^ { 3 } \frac { \partial } { \partial x ^ { 1 } } \otimes \left(d x^{2} \otimes\right.\right. & \left.\left.d x^{3}+d x^{3} \otimes d x^{2}\right)\right)_{X}\left(Y_{1}, Y_{2}\right) \\
& =X^{2} X^{3}\left(c^{\prime \prime}\left(1, Y_{1}^{2}, Y_{2}^{3}\right)+c^{\prime \prime}\left(1, Y_{1}^{3}, Y_{2}^{2}\right)\right) .
\end{aligned}
$$

If $\phi: \mathbb{R}^{n} \ni x \mapsto\left(\frac{x^{1}}{1+\left(x^{3}\right)^{2}}, x^{2}, \ldots, x^{n}\right) \in \mathbb{R}^{n}$ and $t=\frac{\partial}{\partial x^{1}} \otimes d x^{2} \otimes d x^{2}$, then $\phi^{*} t=\left(1+\left(x^{3}\right)^{2}\right) \frac{\partial}{\partial x^{1}} \otimes d x^{2} \otimes d x^{2}$. Taking $p=1,3(3$ before 1$)$ and these $\phi$ and $t$ in (20) and combining this with (70) and (72) respectively we get

$$
L_{\mathbb{R}^{n}}^{\prime \prime \prime}\left(\left(x^{3}\right)^{2} \frac{\partial}{\partial x^{1}} \otimes d x^{2} \otimes d x^{2}\right)_{X}\left(Y_{1}, Y_{2}\right)=\left(X^{3}\right)^{2} c^{\prime \prime}\left(1, Y_{1}^{2}, Y_{2}^{2}\right) .
$$

From (83) and (20) with $p=1, \phi: \mathbb{R}^{n} \ni x \mapsto\left(x^{1}, x^{3}, x^{2}, x^{4}, \ldots, x^{n}\right) \in \mathbb{R}^{n}$ and $t=\left(x^{3}\right)^{2} \otimes \frac{\partial}{\partial x^{1}} \otimes d x^{2} \otimes d x^{2}$ it follows that

$$
L_{\mathbb{R}^{n}}^{\prime \prime \prime}\left(\left(x^{2}\right)^{2} \frac{\partial}{\partial x^{1}} \otimes d x^{3} \otimes d x^{3}\right)_{X}\left(Y_{1}, Y_{2}\right)=\left(X^{2}\right)^{2} c^{\prime \prime}\left(1, Y_{1}^{3}, Y_{2}^{3}\right) .
$$

From (83), (82), (84), (70) and (20) with $p=1, U=\left\{x \in \mathbb{R}^{n}: x^{3} \neq 0\right\}$, $\phi: U \ni x \mapsto\left(x^{1}, x^{2} x^{3}, x^{3}, \ldots, x^{n}\right) \in \mathbb{R}^{n}$ and $t=\frac{\partial}{\partial x^{1}} \otimes d x^{2} \otimes d x^{2}$ it follows that

$$
\begin{aligned}
\left(X^{3}\right)^{2} c^{\prime \prime}\left(1, Y_{1}^{2}, Y_{2}^{2}\right)+X^{2} & X^{3}\left(c^{\prime \prime}\left(1, Y_{1}^{2}, Y_{2}^{3}\right)\right. \\
& \left.+c^{\prime \prime}\left(1, Y_{1}^{3}, Y_{2}^{2}\right)\right)+\left(X^{2}\right)^{2} c^{\prime \prime}\left(1, Y_{1}^{3}, Y_{2}^{3}\right) \\
& =c^{\prime \prime}\left(1, X^{3} Y_{1}^{2}+X^{2} Y_{1}^{3}, X^{3} Y_{2}^{2}+X^{2} Y_{2}^{3}\right),
\end{aligned}
$$

because $\phi^{*} t=\left.\frac{\partial}{\partial x^{1}} \otimes\left(x^{3} d x^{2}+x^{2} d x^{3}\right) \otimes\left(x^{3} d x^{2}+x^{2} d x^{3}\right)\right|_{U}$. From (85) we see that for all $w, x, y, z \in A$,

$$
w x c^{\prime \prime}(1, y, z)=c^{\prime \prime}(1, w y, x z) \text {. }
$$

Define $P=c^{\prime \prime}(1,1,1)$. Of course, $P \in A$. By (2),

$$
\bar{P}_{\mathbb{R}^{n}}^{p}\left(x^{2} \frac{\partial}{\partial x^{1}} \otimes d x^{1} \otimes d x^{1}\right)_{X}\left(Y_{1}, Y_{2}\right)= \begin{cases}P X^{2} Y_{1}^{1} Y_{2}^{1} & \text { if } p=1, \\ 0 & \text { if } p \in\{2, \ldots, n\} .\end{cases}
$$

From (86) and (78) it follows that $x y z P=c^{\prime \prime}(x, y, z)$ for all $x, y, z \in A$. Combining this with (87) gives

$$
\begin{aligned}
\bar{P}_{\mathbb{R}^{n}}^{p}\left(x^{2} \frac{\partial}{\partial x^{1}} \otimes d x^{1} \otimes d x^{1}\right)_{X} & \left(Y_{1}, Y_{2}\right) \\
& = \begin{cases}c^{\prime \prime}\left(X^{2}, Y_{1}^{1}, Y_{2}^{1}\right) & \text { if } p=1, \\
0 & \text { if } p \in\{2, \ldots, n\} .\end{cases}
\end{aligned}
$$

From (88) and the lemma we see that (77), (66) and (63) lead to $L^{\prime \prime}=\bar{P}$. Analyzing (44), (46), (48), (57), (59) and (87), one easily finds that $R, S$, $S^{\prime}, Q, Q^{\prime}$ and $P$ are uniquely determined, which completes the proof. 


\section{References}

[1] J. Dębecki, Linear liftings of p-forms to q-forms on Weil bundles, Monatsh. Math. 148 (2006), 101-117.

[2] J. Gancarzewicz, W. Mikulski and Z. Pogoda, Lifts of some tensor fields and connections to product preserving functors, Nagoya Math. J. 135 (1994), 1-41.

[3] I. Kolář, P. W. Michor and J. Slovák, Natural Operations in Differential Geometry, Springer, 1993.

Instytut Matematyki

Uniwersytet Jagielloński

Reymonta 4

30-059 Kraków, Poland

E-mail: debecki@im.uj.edu.pl

Received 13.10.2006

and in final form 2.3.2007 